\title{
Better care for older people with hip fractures: a call for action and papers!
}

\author{
Anette Hylen Ranhoff ${ }^{1}$
}

Published online: 30 April 2019

(c) The Author(s) 2019

Fragility fractures, and particularly hip fractures, are common among older people, and the prognosis is serious in terms of mobility, independence in daily life activities and cognition with almost half of the patients never achieving the same function as before the fracture [1]. The hip fracture patients have two-threefold excess mortality [2], highest during the first month and year, which is mainly due to comorbidities and complications affecting the frail and multimorbid patients [3]. The typical hip fracture patient is a woman who is more than 80 years old with pre-fracture chronic diseases, frailty, osteoporosis, sarcopenia and often also has cognitive impairment. In other words; these patients are typical geriatric patients. Complications are not limited to orthopedic complications, but infections, thromboembolic events, anemia, exacerbations of cardiac and pulmonary disease, and geriatric syndromes such as delirium, malnutrition, pressure ulcers and new falls are frequent. The patients from long-term-care are particularly prone to bad outcomes from hip fractures, but are mostly excluded from research aiming to improve care.

To improve outcomes and prognosis for older people with hip fractures, orthogeriatric care has been developed. Orthogeriatric care consists of the best from orthopedic and geriatric care given by an interdisciplinary team and has been proved to be effective [4].

One consequence of the aging of society is an enormous increase in fragility fracture incidence which imposes a massive burden on patients, their families, health systems and societies globally. At the same time, it is growing evidence for how to better care for older people with hip fractures and other fragility fractures, regarding models of care and care elements, improved anesthetic and surgical methods, as well as secondary prevention. Despite these advances in evidence for good care there are many unsolved problems,

Anette Hylen Ranhoff

Anette.ranhoff@uib.no

1 University of Bergen, Bergen, Norway and development and implementation of optimal care is a challenge in many hospitals throughout Europe as well as in the rest of the world.

This is the background for the newly published "Global Call to Action to Improve the Care of People with Fragility Fractures", initiated by the Fragility Fracture Network (FFN) (fragilityfracturenetwork.org). Several leading organizations, also the EuGMS, agreed that a global multidisciplinary and multiprofessional collaboration, resulting in a Global Call to Action ( $\mathrm{CtA}$ ), would be the right step to improve the care of people presenting with fragility fractures [5].

On this line, this journal (EGM) and the Journal of the American Geriatrics Society (JAGS) have agreed to launch a joint initiative on orthogeriatrics. The aims of our initiative are to present an overview of the newest evidence for good orthogeriatric care, raise important research questions to initiate progress in this important research area, and finally to present arguments for implementation and how to implement evidence-based care in different health services for older people with fragility fractures.

Many groups around the world are publishing on orthogeriatric care, and the clinical challenges are numerous. We are inviting papers looking at how care is delivered and what are the outcomes, as well as topics such as anesthesia, anemia, delirium, infections, rehabilitation, osteoporosis, sarcopenia, malnutrition, emotional problems - all related to older patients with hip fractures. Systematic and narrative reviews are particularly welcome.

EGM and JAGS will divide papers according to geographical areas. JAGS will call for and publish papers from the Northern America (US and Canada) and Asia (https://onlin elibrary.wiley.com/doi/10.1111/jgs.15917), while EGM will welcome papers from Europe and the rest of the world (Middle-East, Africa, Oceania and Latin- and South America).

Finally, I encourage geriatricians and other health professionals to take action for better care for older people with hip fracture-do research and send it for publication in EGM! 


\section{Compliance with ethical standards}

Conflict of interest The author declares that she has no conflict of interest.

Ethical approval This article does not contain any studies with human participants or animals performed by any of the authors.

Informed consent For this type of study formal consent is not required.

Open Access This article is distributed under the terms of the Creative Commons Attribution 4.0 International License (http://creativeco mmons.org/licenses/by/4.0/), which permits unrestricted use, distribution, and reproduction in any medium, provided you give appropriate credit to the original author(s) and the source, provide a link to the Creative Commons license, and indicate if changes were made.

\section{References}

1. Dyer SM, Crotty M, Fairhall N, Magaziner J, Beaupre LA, Cameron ID, Sherrington C, Fragility Fracture Network (FFN) Rehabilitation Research Special Interest Group (2016) A critical review of the long-term disability outcomes following hip fracture. BMC Geriatr. 16:158
2. Katsoulis M, Benetou V, Karapetyan T, Feskanich D, Grodstein F, Pettersson-Kymmer U et al (2017) Excess mortality after hip fracture in elderly persons from Europe and the USA: the CHANCES project. J Intern Med 281(3):300-310

3. Smith T, Pelpola K, Ball M, Ong A, Myint PK (2014) Pre-operative indicators for mortality following hip fracture surgery: a systematic review and meta-analysis. Age Ageing 43(4):464-471

4. Prestmo A, Hagen G, Sletvold O, Helbostad JL, Thingstad P, Taraldsen $\mathrm{K}$ et al (2015) Comprehensive geriatric care for patients with hip fractures: a prospective, randomised, controlled trial. Lancet 385(9978): 1623-1633

5. Dreinhöfer KE, Mitchell PJ, Bégué T, Cooper C, Costa ML, Falaschi P, on behalf of: the Fragility Fracture Network (FFN); European Geriatric Medicine Society (EuGMS); European Federation of National Associations of Orthopaedics and Traumatology (EFORT); International Collaboration of Orthopaedic Nursing (ICON); International Geriatric Fracture Society (IGFS); International Osteoporosis Foundation (IOF) et al (2018) A global call to action to improve the care of people with fragility fractures. Injury 49(8):1393-1397

Publisher's Note Springer Nature remains neutral with regard to jurisdictional claims in published maps and institutional affiliations. 